\section{One Degree Micro-Macro Manipulator Integration Test}

\section{Richard J. Norcross}

U. S. DEPARTMENT OF COMMERCE

Technology Administration Intelligent Systems Division National Institute of Standards and Technology

Gaithersburg, MD 20899

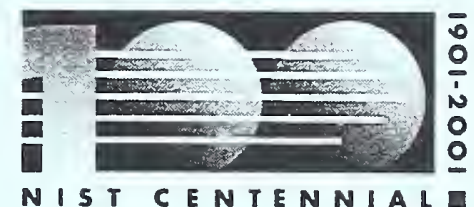

NIST CENTENNIALE

\section{NIST}

National Institute of Standards and Technology

Technology Administration

U.S. Department of Commerce

.1556

N0.6562

$2000<2$ 



\section{NISTIR 6562}

\section{One Degree Micro-Macro Manipulator Integration Test}

\section{Richard J. Norcross}

U. S. DEPARTMENT OF COMMERCE Technology Administration Intelligent Systems Division National Institute of Standards and Technology

Gaithersburg, MD 20899

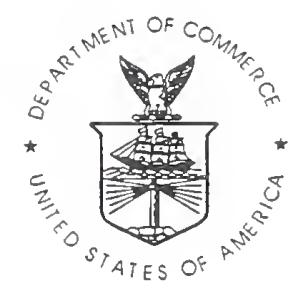

U.S. DEPARTMENT OF COMMERCE Norman Y. Mineta, Secretary

TECHNOLOGY ADMINISTRATION Dr. Cheryl L. Shavers, Under Secretary of Commerce for Technology

NATIONAL INSTITUTE OF STANDARDS AND TECHNOLOGY

Raymond G. Kammer, Director 


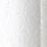




\title{
ONE DEGREE MICRO-MACRO MANIPULATOR INTEGRATION TEST
}

\author{
For the \\ Automated Paint Application, Containment and Treatment System \\ (APACTS)
}

\author{
for: \\ Naval Surface Warfare Center \\ Carderock Division \\ 9500 MacArthur Blvd. \\ West Bethesda, MD 20817-5700
}

Richard J. Norcross

Intelligent Systems Division

National Institute of Standards and Technology

Gaithersburg, MD 20899-8230 


\section{Disclaimer}

No approval or endorsement of any commercial product by the National Institute of Standards and Technology is intended or implied. Certain commercial equipment, instruments, or materials are identified in this report to facilitate understanding. Such identification does not imply recommendation or endorsement by the National Institute of Standards and Technology, nor does it imply that the materials or equipment identified are necessarily the best available for the purpose.

\section{Copyright}

This publication was prepared by United States Government employees as part of their official duties and is, therefore, a work of the U.S. Government and not subject to copyright.

\section{Ackmowledgement}

This report is partial fulfillment of sub-contract AM 02-9802001 with AmDyne Corporation of Millersville, Maryland. 


\section{Abstract}

The Carderock Division of the Naval Surface Warfare Center is developing the Automated Paint Application, Containment, and Treatment System (APACTS) to apply anticorrosive and anti-fouling paints in an environmentally sound manner. To provide accurate motion over a very large volume, the APACTS motion system employs a selfpropelled base carrying a long reach macro-manipulator which in turn carries a quick response micro-manipulator to maneuver the paint nozzle and containment device along the ship hull. The manipulators run separate but coordinated trajectories whose combination is the path of the paint nozzle. Based on sensors and feedback from the operator's observations, the micro-manipulator's trajectory is shifted to keep the paint nozzle at the appropriate position relative to the surface being painted. The micromanipulator communicates the shift to the macro-manipulator, which adjusts its trajectory to remove the error and re-center the micro-manipulator. This report investigates the minimum requirements for the interface between the macro and micromanipulators. The investigation includes experiments that test the micro-macro interface by observing the system 's response to induced errors. The results indicate an interface reporting the size of the shift and the frequency of the report is sufficient to control the manipulator system, but may not be sufficient for the APACTS application. The report includes possible improvements to the interface. 



\section{DRAFT}

\section{Table of Contents}

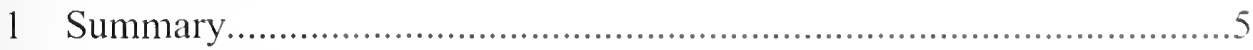

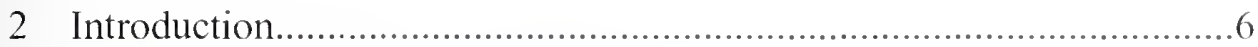

3 Methods, Assumptions, and Procedures............................................

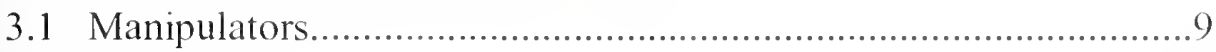

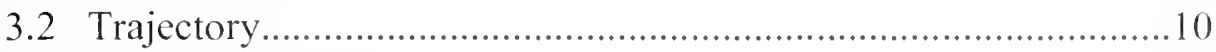

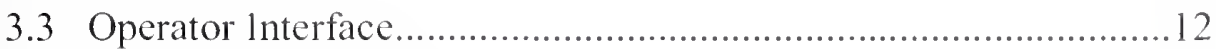

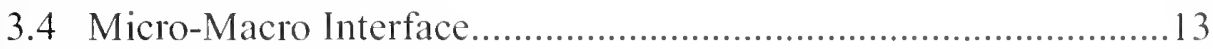

4 Results and Discussions................................................................. 14

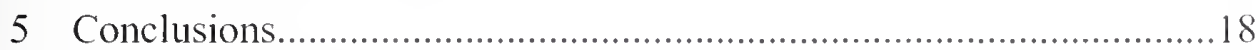

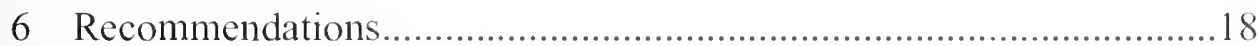

6.1 Continuous Feedback .............................................................

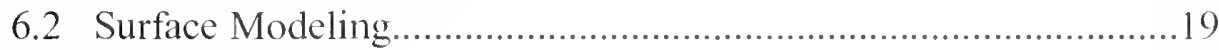

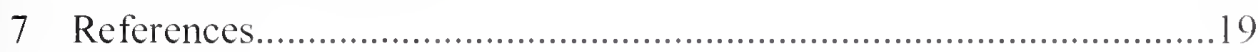

\section{List of Figures}

Figure 1. APACTS System Concept......................................................

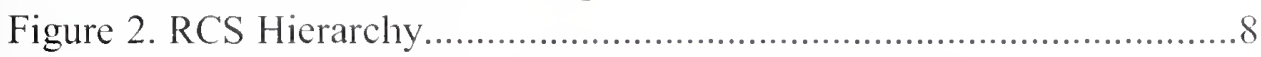

Figure 3. Modified ATR-60 AWP as Macro-Manipulator........................9

Figure 4. Micro-Manipulator on AWP Basket........................................10

Figure 5. Micro Manipulator Trajectory..................................................11

Figure 6. Trajectory Speeds to Simulate Curved Surface Adjustments.....12

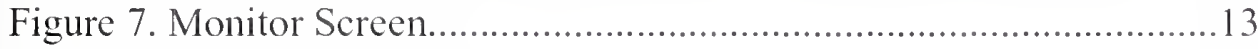

Figure 8. Sample Test Run................................................................... 15

Figure 9. Correction with Initial Speed Error.............................................. 16

Figure 10. Correction with Initial Position Error.........................................16

Figure 11. Simulated Surface Following.................................................17

Figure 12. Relocation Sequence with Excess Boom Speed...........................18 



\section{Summary}

The Cardcrock Division of the Naval Surface Warfare Ccnter is developing the Automated Paint Application, Containment, and Treatment System (APACTS) to apply anti-corrosive and anti-fouling paints onto Navy ship hulls in an environmentally sound manner. APACTS' motion system (Figure 1) employs three motion components; a selfpropelled, repositionable base, a long reach macro-manipulator, and a quick response micro-manipulator to move the paint nozzle and containment device along the hull surface. The combined motion trajectories of the macro and micro-manipulators form the paint application trajectory. This report investigates the interaction between these two manipulators.

\section{Figure 1. APACTS System Concept}

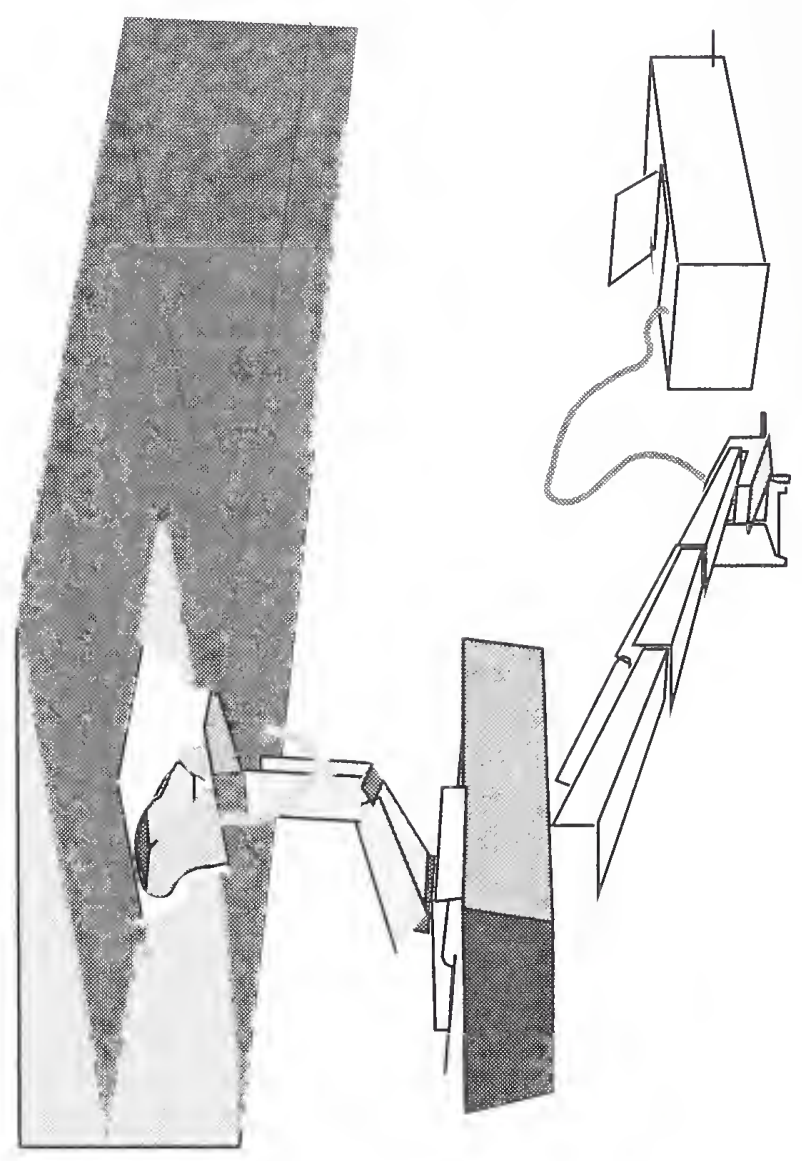



The micro-manipulator trajectory is a repetitive sequence of positions that traverses only a portion of the micro-manipulator's range in any direction. That portion of the micromanipulator's volume that is not used by the trajectory is called the excess volume. The operator removes nozzle position errors by shifting the micro-manipulator's trajectory into the excess volume. For example, if the micro-manipulator can reach between 10 and $30 \mathrm{~cm}$ in a given direction, and the trajectory requires only $10 \mathrm{~cm}$ in that direction, then a nozzle position error of $3 \mathrm{~cm}$ can be removed by having the micro-manipulator's trajectory operate between 18 and $28 \mathrm{~cm}$ rather then between 15 and $25 \mathrm{~cm}$. The micromanipulator periodically sends the accumulated shifts (i.e., the offset) to the macromanipulator. The offset triggers a change in the macro-manipulator trajectory that reverses the position errors and results in the micro-manipulator being re-centered.

To test the system's ability to coordinate macro and micro motions in response to corrective inputs, experiments were conducted where two types of known errors were introduced into the macro manipulator's trajectory. In the first set of tests, the macromanipulator motion begins a small distance from the intended start position to produce a tool position error. In separate tests, changes to the macro-manipulator's velocity, either in the beginning or middle of the run, produce a tool speed error. The experiments verify the interfaces are sufficient to coordinate and stabilize the two manipulators. However, the minimum micro-macro interface suffers periodic position errors that may cause gaps in the paint coverage and be unacceptable for the APACTS application.

A review of the test results indicate the overall system performance may be improved through enhancements to the detection and communication interfaces. In these experiments, an operator detects the position error. The operator was unable to observe the proper nozzle position through much of the micro-manipulator's trajectory cycle. Speed errors during this time accumulate into significant position errors. Improvements in position detection, either through improved operator assistance or through an automatic sensor, may avoid error accumulation and improve overall system performance. The micro-macro interface used in these experiments does not provide the macro-manipulator with the curvature of the surface. Since surface curvature affects the required macromanipulator speed, curvature data may allow the macro-manipulator to more accurately and rapidly adjust its velocity in response to curvature changes. The value of these enhancements must be verified by separate tests.

\section{Introduction}

To guard against the harshness of the sea, ships are covered with anti-corrosive and antifouling paints that must be periodically replaced to maintain their effectiveness. During replacement, hazardous airborne particles (HAPs) are inadvertently discharged into the environment diminishing air quality, and endangering shipyard personnel and the 

surrounding harbor. The Carderock Division, Naval Surface Warfare Center (CD-NSWC), Environmental Quality Department conducts research and development leading to fleet implementation of pollution-control materials, processes, and equipment that enable Navy ships to be environmentally responsible. CD-NSWC is responsible for providing the Navy with the technical expertise to solve existing and emerging waste management problems. Pursuant to that responsibility, CD-NSWC is developing the Automated Paint Application, Containment, and Treatment System (APACTS) to significantly reduce HAP discharge from the painting operation [1].

The primary components of APACTS are the delivery system, the containment system, the treatment system, and the manipulation system. The delivery system consists of a paint mixer, strainer, sprayer, nozzle, and associated equipment. The containment system surrounds the paint sprayer and includes a capture shroud, recovery vacuum, hoses, and controls. The treatment system includes waste transport, waste isolation, filter elements, and their support equipment. The manipulation system consists of those devices that move the sprayer and containment shroud. The components complement each other to produce an effective, economic, and environmentally-sound system.

The Intelligent Systems Division of the National Institute of Standards and Technology (NIST-ISD) supports APACTS development through investigation of new and existing technologies to carry, maneuver, and manipulate the APACTS sprayer and containment system. Since a single manipulator would be unable to achieve the performance requirements at an acceptable cost, APACTS uses a series of three manipulators to position the system about the dry-dock, to reach along the hull, and to maintain proper standoff and motion. After the mobile base positions APACTS in or around the drydock, a long reach, but slow response macro-manipulator carries a high accuracy, fast response micro-manipulator to simultaneously provide sufficient reach and accuracy.

The combination of dissimilar manipulators is known by several names including; macromicro, macro/micro, maxi-mini, and major-minor. Many researchers have investigated macro-micro control ([2]-[8]). These approaches rely primarily on either a well-defined trajectory or a well modeled pair of manipulators. While none adequately address problems of working throughout a very large volume in a poorly defined environment, several micro-macro control strategies may be extensible to the APACTS problem.

The proposed APACTS controller follows the hierarchical control theory of the Realtime Control system (RCS) [9]. Under RCS, tasks are spatially decomposed along the branches of the hierarchy and temporally decomposed across the levels of the hierarchy. Each controller node contains a Sensor Processing module (SP) that interprets sensor data, a World Model module (WM) that maintains the model of the control system's environment used to plan task sequences, and a Behavior Generation module (BG) that plans and executes the task sequence (Figure 2). An RCS controller treats the 

manipulators as independent systems with a common supervisor that coordinates the subordinates through the initiation of related commands. Cooperation within a task occurs via the hicrarchy's World Model. Thus, to the macro-manipulator, the micromanipulator is effectively a pre-processed sensor. The micro-manipulator"s position within its work volume is an offset value that the macro-manipulator subsequently minimizes with changes to its own motion. As the offset value diminishes, the micromanipulator returns to the center of its volume.

\section{Figure 2. RCS Hierarchy}

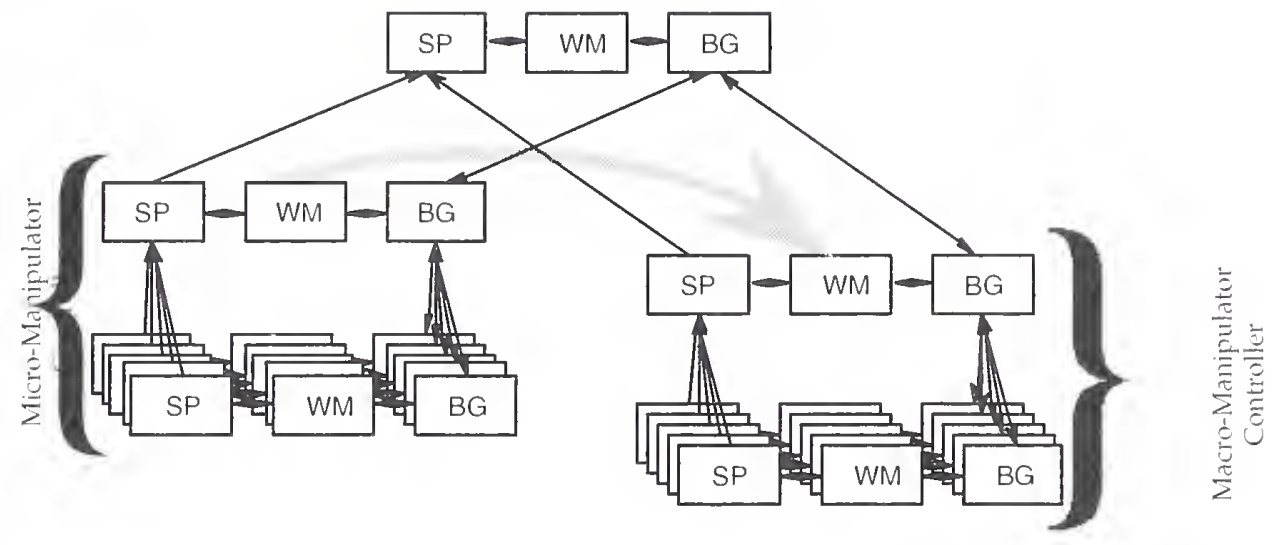

This report reviews the interaction between the macro and micro manipulators. The work here intends to establish sufficient requirements for an interface between two serially linked and cooperating manipulators. We demonstrate that two manipulators, with large differences in their servo frequencies, can be coordinated through an interface operating at a lower frequency then the slower manipulator.

\section{Methods, Assumptions, and Procedures}

The experiment tests the coordination of two serially linked manipulators. The two manipulators execute separate trajectories that, when simultaneously executed, step and maintain a tool point over periodic marks on a vertical surface. We introduce position and velocity errors to the macro-manipulator trajectory to evaluate the system's ability to correct the errors through the macro-micro interface. By way of a camera on the micromanipulator, an operator observes the effect of the error on the tool position and, via a joystick, offsets the micro-manipulator's trajectory to compensate. The micro- 

manipulator communicates the offsets through the micro-macro interface and the macromanipulator adjusts its trajectory to re-center the micro-manipulator.

\subsection{Manipulators}

The macro-manipulator is an ATR-60 aerial work platform (AWP) from Snorkel, Inc. of St. Joseph, MO. The standard AWP has digital proportional valves on several of its actuators. The experiment's AWP has similar digital proportional valves on all actuators and each actuator is fitted with absolute position and relative motion sensors. Servo control modules monitor the actuator motion and adjust the oil flow through the valves to cause the actuator to follow a motion path. A supervisory controller coordinates the actions of the servo modules such that the AWP's basket can follow Cartesian paths or a surface as shown in Figure 3. The macro-manipulator's supervisory controller updates the goal position at $8 \mathrm{~Hz}$. The actuator controllers close the actuator servo loop at $30 \mathrm{~Hz}$.

\section{Figure 3. Modified ATR-60 AWP as Macro-Manipulator}

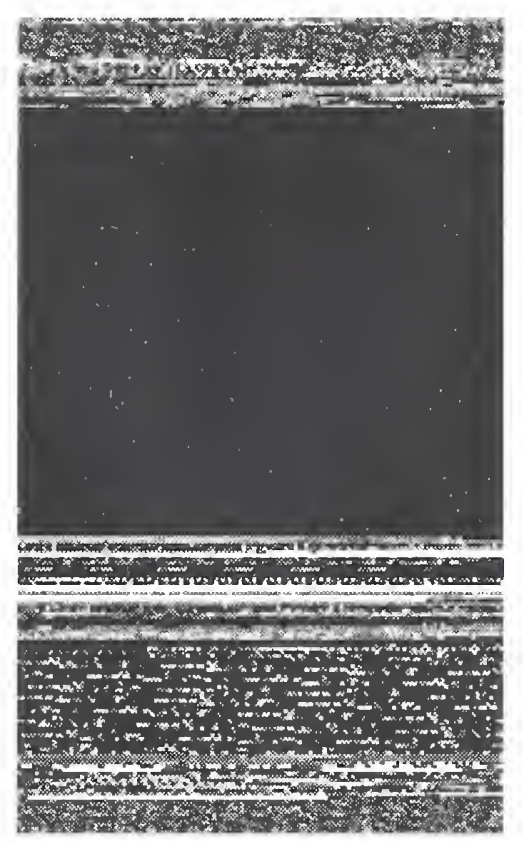

The micro-manipulator in the experiment is a one axis linear actuator carrying a small video camera (Figure 4). The camera provides position feedback to the operator during the experiment. The manipulator is controlled by a Smart Motor from Anamatics, Inc. of Carlsbad, CA. The Smart Motor runs in Anamatics' extended cam mode and contains a repeating trajectory. The controller shifts (or offsets) the trajectory in response to signals on an analog input port. The micro-manipulator controller updates the goal position at $200 \mathrm{~Hz}$ and closes the motor's servo loop at $4 \mathrm{kHz}$. 
Figure 4. Micro-Manipulator on AWP Basket

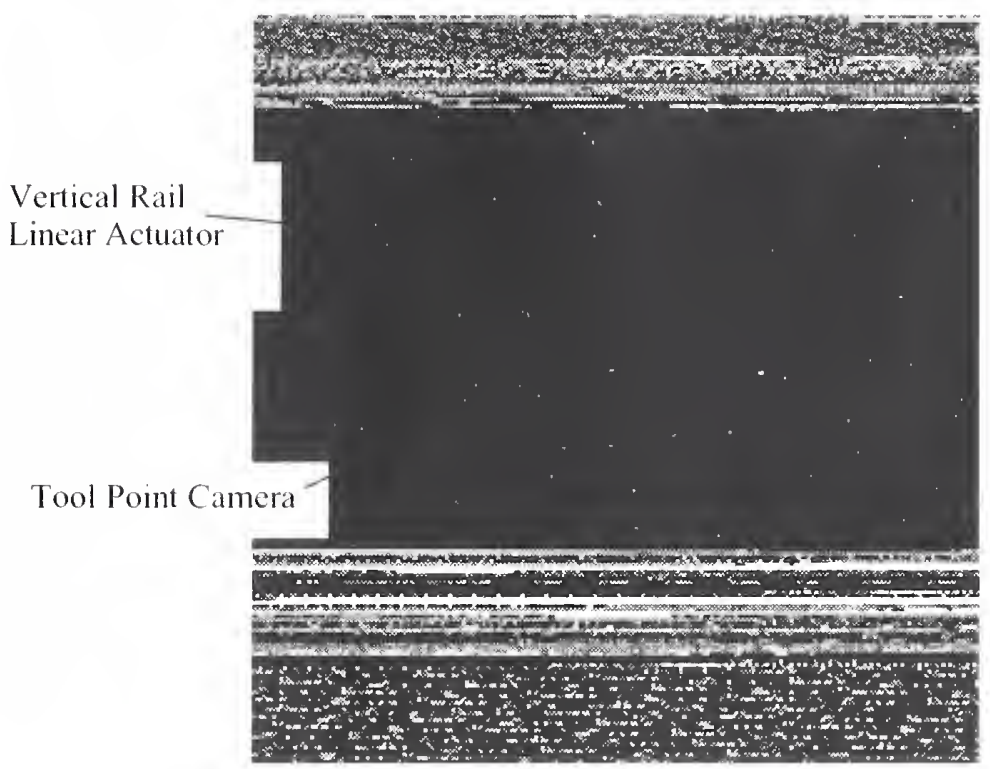

\subsection{Trajectory}

The experiment uses the vertical component of the vertical compensation trajectory discussed in [10]. Under the vertical compensation trajectory, APACTS paints in vertical swaths, where a swath is a set of horizontal stripes painted sequentially from top to bottom. The test trajectory imitates an APACTS application with a $30 \mathrm{~cm}$ (12 in) effective spray width (aligned vertically), a $36 \mathrm{~cm} / \mathrm{s}$ (14 in/s) nozzle speed (applied horizontally), and $240 \mathrm{~m}^{2} / \mathrm{h}\left(2600 \mathrm{ft}^{2} / \mathrm{h}\right)$ production rate. The nominal macro-manipulator trajectory moves the basket vertically down a wall at $5 \mathrm{~cm} / \mathrm{s}(2 \mathrm{in} / \mathrm{s})$. In order to maintain the vertical position during paint application, the nominal micro-manipulator trajectory moves up $19 \mathrm{~cm}(7.5 \mathrm{in})$ for 3.75 seconds $(5 \mathrm{~cm} / \mathrm{s}(2 \mathrm{in} / \mathrm{s})$ ) (the upstroke) then returns to the initial position in the subsequent 2.25 seconds (the relocation stroke). The first loop begins with a $3 \mathrm{~cm} / \mathrm{s}^{2}$ acceleration (Figure 5 ).

Known errors injected into the macro-manipulator trajectory test the system's stability. The error conditions include an initial position error and an initial velocity error. A simulated surface with abrupt curvature changes tests the system through a sequence of sudden velocity errors. 


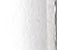




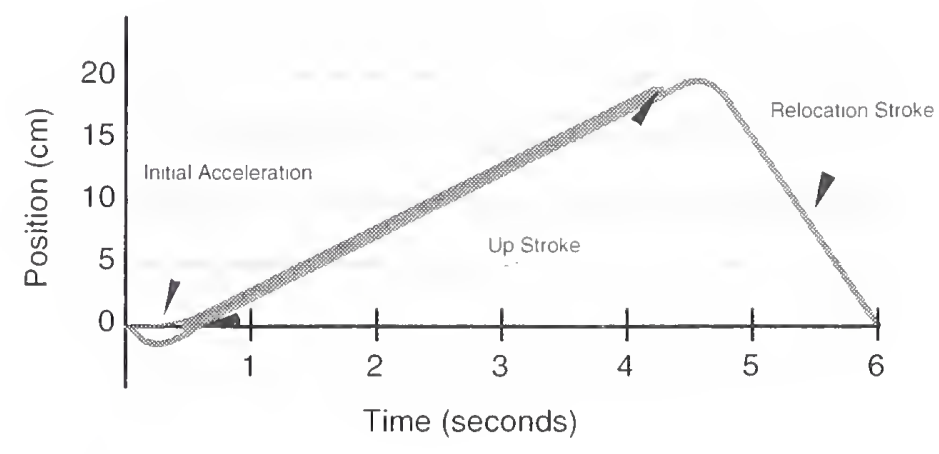

Boom speed modifications simulate changes to the surface curvature. Along a flat surface, the micro-manipulator, whose reference is on the surface, and the macro-manipulator, whose reference is some distance off the surface, move at the same speed. However along convex surfaces, the macro-manipulator must move faster to maintain the proper relative position. Thus abrupt boom speed changes simulate an abrupt shift from a flat surface to a curved surface. With a $183 \mathrm{~cm}$ ( 72 inch) surface radius and a $91 \mathrm{~cm}$ (36 in) standoff, the speed change is $50 \%$ of the nominal speed. To follow an actual wall transiting from a flat to a convex surface, the boom speed would increase from $5 \mathrm{~cm} / \mathrm{s}$ to $7.5 \mathrm{~cm} / \mathrm{s}(2 \mathrm{in} / \mathrm{s}$ to 3 $\mathrm{in} / \mathrm{s})$. To simulate this action, the boom speed is reduced $2.5 \mathrm{~cm} / \mathrm{s}(1 \mathrm{in} / \mathrm{s})$. Similarly, a boom speed increase from $5 \mathrm{~cm} / \mathrm{s}$ to $7.5 \mathrm{~cm} / \mathrm{s}(2 \mathrm{in} / \mathrm{s}$ to $3 \mathrm{in} / \mathrm{s})$ simulates a transition from a flat surface to a concave surface. Similar boom speed changes simulate other transitions.

The simulated test surface (Figure 6) is flat for $61 \mathrm{~cm}$ (24 inches), convex (at $183 \mathrm{~cm}$ radius) for $144 \mathrm{~cm}$ ( $56.5 \mathrm{in}$ ), flat for $30 \mathrm{~cm}$ ( 12 inch), concave (at $183 \mathrm{~cm}$ radius) for 144 $\mathrm{cm}$, and finishes as a flat surface. The transitions generate abrupt $2.5 \mathrm{~cm} / \mathrm{s}$ changes in the boom speed which create position errors that are removed by the operator's input. The anticipated changes for an APACTS application are less severe then those used in the experiment. 



\section{Figure 6. Trajectory Speeds to Simulate Curved Surface Adjustments}

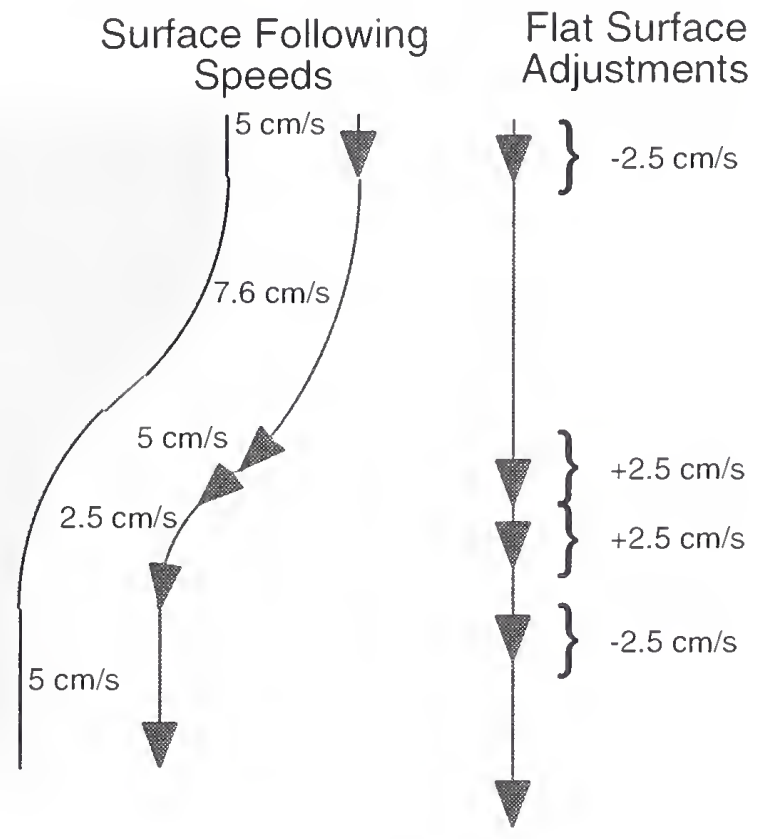

\subsection{Operator Interface}

The macro-micro control concept relies on observations of the task to determine part of the motions of the micro-manipulator. Since the position based on actuator sensors and kinematics calculation is unreliable, other sensors must detect the relative pose of the tool point to the task. While the full APACTS application may use automatic sensors to generate the information, an operator generates the appropriate feedback signals for this experiment.

The experiment's operator interface consists of the video signal from the micromanipulator's camera and a one degree of freedom joystick. The operator observes the effective motion along the surface and modifies the micro-manipulator's trajectory to remove position errors. The micro-manipulator controller rejects trajectory modifications during periods when the operator is unable to view the proper nozzle position.

Under the vertical compensation trajectory, APACTS paints in vertical swaths, where a swath is a set of horizontal stripes painted sequentially from top to bottom. A stripe width is the difference between the width of the paint spray and the overlap between stripes. The experiment's test surface is marked at $30 \mathrm{~cm}$ (12 in) intervals with horizontal lines that represent the lower edges of the paint stripes. The micro-manipulator carries a small camera, whose video signal is displayed on a monitor at the control station. A small 
arrow is attached to the monitor's screen to give the operator a reference to assess the position of the camera relative to the surface (Figure 7). During operation, the arrow would be positioned at the bottom of the previous stripe to provide the proper overlap.

The operator commands a micro-manipulator trajectory shift through a joystick potentiometer connected to the micro-manipulator's controller. The joystick input is scaled to correct the position at up to $7.5 \mathrm{~cm} / \mathrm{s}(3 \mathrm{in} / \mathrm{s})$. Since there is no reference for the visual feedback during the relocation stroke (i.e., because the lines move relative to the arrow), the operator's input modifies the trajectory only during the upstroke portion of the trajectory.

\section{Figure 7. Monitor Screen}

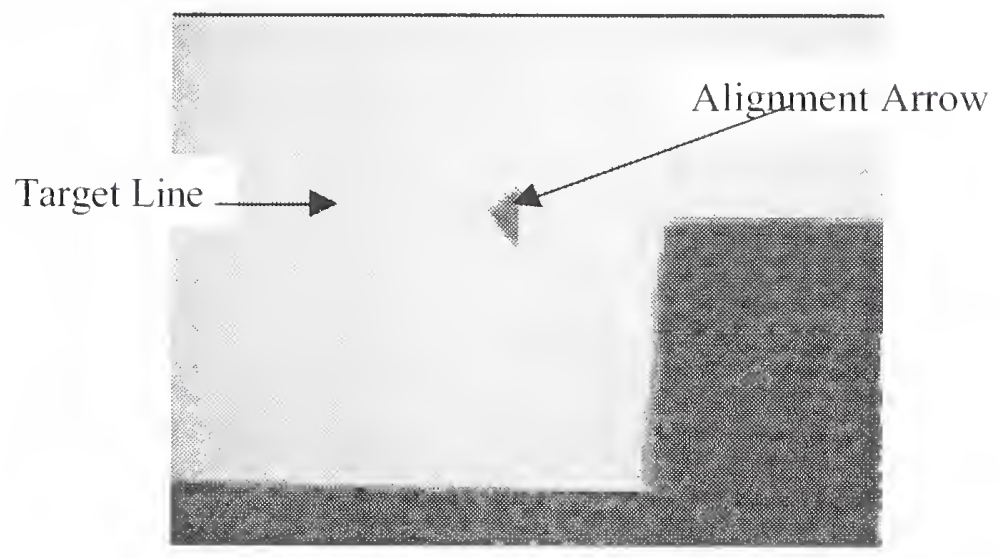

\subsection{Micro-Macro Interface}

The experiments verify a minimally sufficient interface between the micro and macro manipulators. The interface is the information passed between the controllers of the two manipulators and the semantics of that data. The information includes the data exchanged at run-time and any implied data that is encapsulated in the computer code of the transmitting and receiving controllers. The run-time data consists of the offset to the micro-manipulator trajectory generated by the operator interface. The implied data includes the surface direction (always down in the experiment), the data format and the data frequency.

The micro-macro interface connection is a port on a Seriplex bit bus (from Square D, Inc.) that serves as the macro-manipulator's communication and control bus. To fit on the bit bus, the offset is shifted and scaled to an 8 bit value. The scale and offset values, along with the implied units, form the interface's data format and are embedded in software on both sides of the interface. 
The data's frequency and regularity follow the requirements and limitations of the micro and macro manipulators. Data passed across the interface at frequencies greater than the motion control frequency is unusable by the receiving controller. Generally, a rough order of magnitude separates the interface frequency (which transfers processed data) and the motion control frequency (which issues commands). Since the macro-manipulator's hydraulic valves limit the macro-manipulator position command frequency to $8 \mathrm{~Hz}$, the micro-macro interface transfers data at $1.1 \mathrm{~Hz}$ in the experiemnts. However, the micromanipulator is not able to send data every 0.9 seconds. Since the operator doesn't have a visual reference of the proper position relative to the surface during the relocation stroke, the micro-manipulator does not accept offset changes during the relocation stroke and there are no reports during those 2.25 seconds. Thus the data pattern consists of five evenly spaced reports within 3.75 seconds followed by no data for 2.25 seconds. The interpretation of the offset transmission pattern is embedded in the macro-manipulator software.

The macro-manipulator controller modifies the boom speed based on the offset data. The controller scales the current offset, and adds a scaled running sum of previous offsets to produce the new surface following speed. The controller updates the running sum only once per micro-manipulator loop to ensure a consistent interval. The macro-manipulator's acceleration limits smoothly apply the newly computed boom speed. These limits were not varied during the experiment.

The micro-macro interface transfers the micro-manipulator trajectory offset and trajectory state. The macro-manipulator controller adjusts the boom speed based on the data and encoded knowledge of the data format and the data frequency and regularity.

\section{Results and Discussions}

The experiments test the macro-micro interface by observing the system's response to controlled errors. While the system's performance also depends on the operator's and the macro-manipulator's responsiveness, the system response did not need to be optimal to test the sufficiency of the micro-macro interface.

The system performance depends greatly on the responsiveness of the operator. A rapid micro-manipulator shift quickly eliminates position errors, produces proper offset values, appropriately adjusts the macro-manipulator speed, and limits the accumulated errors. However, an excessively fast interface can cause the operator to overshoot the proper position and generate erroneous macro-manipulator trajectory changes. The appropriate values for slow, fast, and too fast depend on the personal preferences of the operator. After several trials, the test operator ran the experiments with a maximum correction rate of approximately $7 \mathrm{~cm} / \mathrm{s}(3 \mathrm{in} / \mathrm{s})$. 

The macro-manipulator speed correction follows the operator's maximum correction rate. The macro-manipulator speed correction is $0.5 \mathrm{~cm} / \mathrm{s} / \mathrm{cm}$ for the offset and $0.2(\mathrm{~cm} / \mathrm{s}) / \mathrm{cm}$ for the running sum of the offset. With the $1.1 \mathrm{~Hz}$ interface frequency, the macromanipulator adjusts the boom speed at up to $80 \%$ of the operator's maximum correction rate. Optimal values for the macro-manipulator were not investigated. However, when the macro-manipulator attempted adjustments at over $100 \%$ of the operator's corrections the system became unstable.

All experiments were run by the same operator and with the same macro-manipulator adjustment values. Figure 8 through Figure 11 display the results of representative test runs. These figures show the micro-trajectory offset sent across the interface and the commanded boom speed. The actual boom speed is subject to the macro-manipulator acceleration limits and closely follows the commanded speed.

The target lines on the vertical surface represent the lower end of the paint stripes in an APACTS application. Like the paint stripes the lines are not perfect. Even without intentional errors, the operator makes numerous corrections to keep the camera on target. In Figure 8 many small offsets are closely followed by similar adjustments to the boom speed.

\section{Figure 8. Sample Test Run}

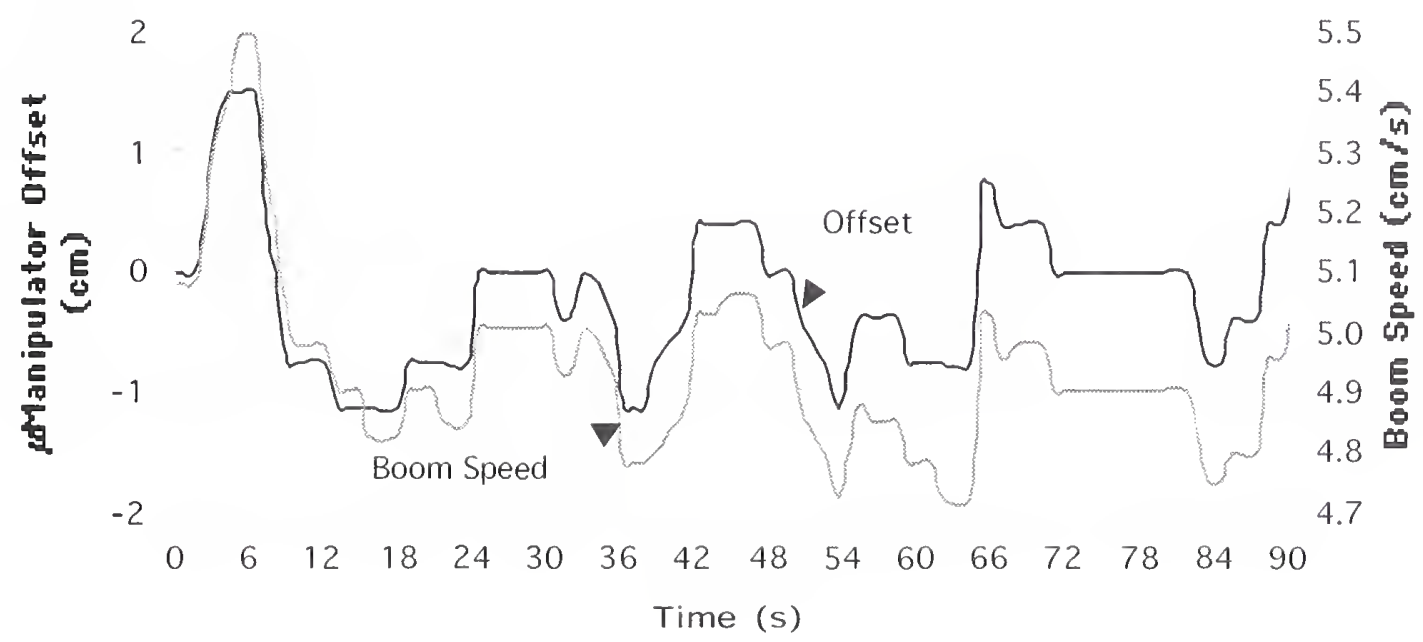

The first error test began the swath with a $2.5 \mathrm{~cm} / \mathrm{s}(1 \mathrm{in} / \mathrm{s})$ boom speed error. The boom speed error quickly generates a position error that is corrected by the operator through offsets to the micro-manipulator's trajectory (Figure 9). In response to the operator's offsets, the macro-manipulator controller decreases the boom speed. The boom speed reaches its nominal value $(5 \mathrm{~cm} / \mathrm{s}, 2 \mathrm{in} / \mathrm{s})$ within approximately one and one-half micro- 

manipulator loops. The resulting offset $(\approx 11 \mathrm{~cm})$ causes the boom to continue to slow to less than the nominal value. The slower boom speed then generates an opposite position error that the operator corrects by reducing the micro-manipulator trajectory offset. The boom speed and the offset slowly return to their nominal values.

Figure 9. Correction with Initial Speed Error

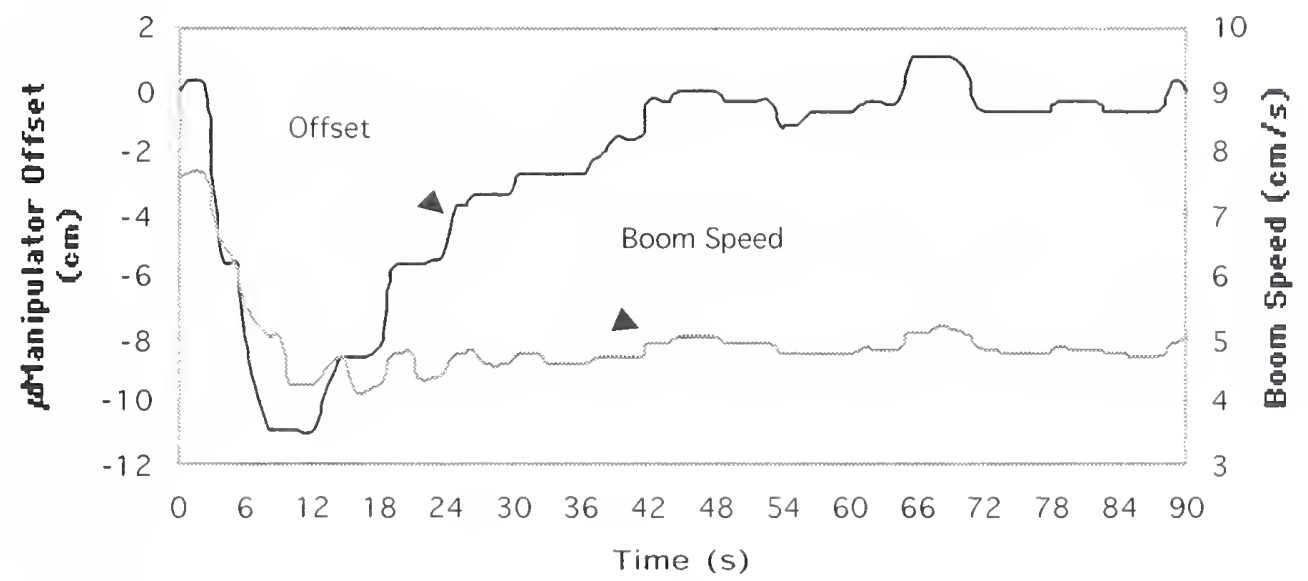

The second error test covers a misalignment between the camera and target at the beginning of the swath. Figure 10 shows the response to an initial $18 \mathrm{~cm}$ ( $7 \mathrm{in}$ ) position error. The operator closes the position error with a similar sized offset within a single micro-manipulator loop. The system then follows the same pattern as shown in Figure 9 except instead of building a large offset, the existing offset is removed.

\section{Figure 10. Correction with Initial Position Error}

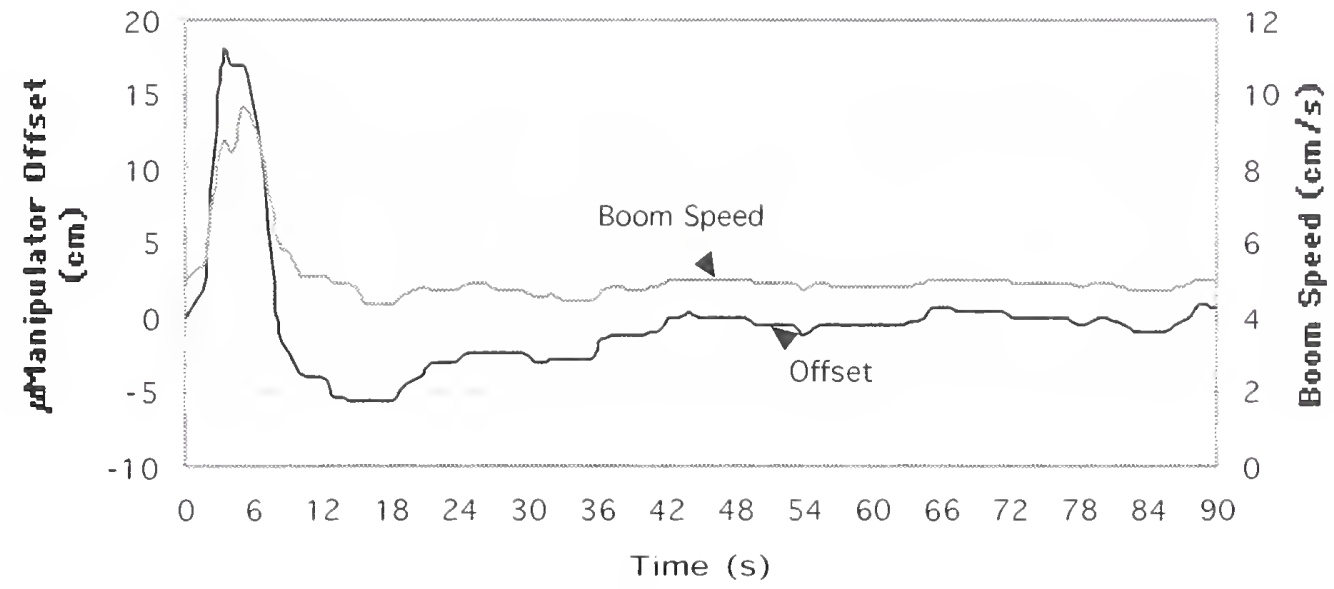

One Degree Micro-Macro Manipulator Integration Test 

A simulated surface experiment shows the control system ability to handle abrupt boom speed errors (Figure 11). An interesting aspect of the micro-macro data interface sequence is seen by comparing the boom speed change at 12 seconds with the one near 72 seconds. The change at 12 seconds occurred at the beginning of the micro-manipulator upstroke and was observed and quickly corrected by the operator. The change near 72 seconds occurred later in the upstroke. The acceleration limit shifts the speed change into the relocation stroke where the operator can not observe the change. The erroneous speed was in effect throughout the relocation stroke and caused an error that is still evident in the micro manipulator offset at the end of the swath (at 90 seconds).

\section{Figure 11. Simulated Surface Following}

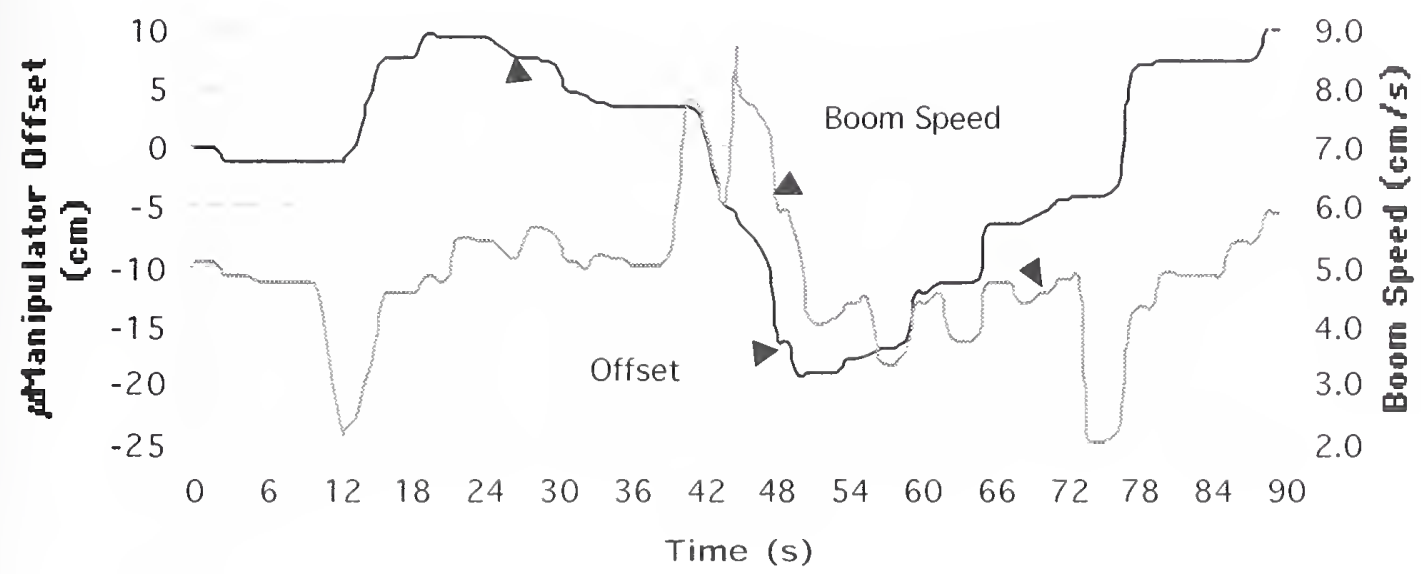

A large offset does not equate to a large position error. However a rapidly changing offset indicates an error correction. The most significant error occurs when a boom speed error existed during the relocation stroke. Since there isn't a surface reference during relocation, the operator can not detect and correct the crror. Figure 12 shows the operator's view during a relocation sequence when the required boom speed changes by $2.5 \mathrm{~cm} / \mathrm{s}(1 \mathrm{in} / \mathrm{s})$ at the beginning of the relocation stroke. Until near the end of the 2.25 second relocation, the operator is unaware of the accumulating error. The final $5 \mathrm{~cm}$ ( 2 in) error takes approximately 0.6 second to correct. The correction could occur in an unpainted horizontal section or could result in up to $20 \mathrm{~cm}$ ( 8 in) of poorly overlapped paint. 

Figure 12. Relocation Sequence with Excess Boom Speed

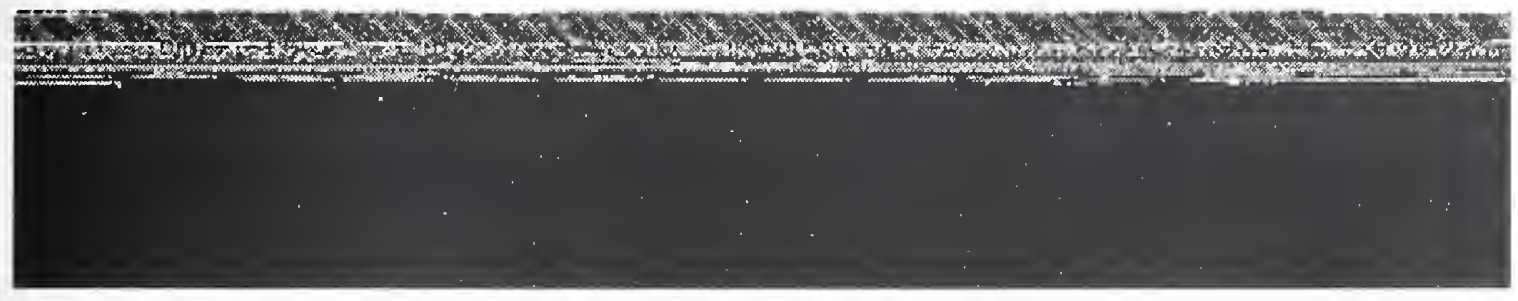

$03.75 \mathrm{~s}$

$04.42 \mathrm{~s}$

$05.28 \mathrm{~s}$

$06.00 \mathrm{~s}$

\section{Conclusions}

A small, quick-response micro-manipulator mounted on a large, sluggish macromanipulator produces a manipulator with large volume and good accuracy. The individual manipulators execute separate trajectories that, when combined, produce a desired tool path on a surface. Induced errors in the large manipulator's trajectory test the system's response to perturbations and errors. The test results demonstrate a very low bandwidth interface can coordinate a low bandwidth (i.e., servo frequency) macro-manipulator with the a high bandwidth (i.e., high servo frequency) micro-manipulator. An interface, consisting of the micro-manipulator's trajectory offset, the time of the offset, and the direction of the offset, along with the data format and transmission pattern, is adequate to maintain control of the system. However, the inability to make corrections during the relocation stroke of the micro-manipulator's trajectory creates errors that may cause coverage gaps that would be unacceptable for the APACTS application.

\section{Recommendations}

The following areas of interface refinement should be investigated. They offer excellent possibilities of improving the APACTS system.

\subsection{Continuous Feedback}

The experiment's setup reported offset positions only on the upstroke of the micromanipulator trajectory. This creates an irregular interval on which to correct the boom speed. More responsive action will likely be possible with regular observations of the trajectory offset. Regular observation would also allow the interface to be more independent of the macro-manipulator's program code.

Regular observations can be accomplished by superimposing a target position on the task position feedback. The observation reference (the alignment arrow in Figure 7) would shift to the next reference mark (e.g. the bottom of the recent paint stripe) on the monitor screen at the start of the relocation stroke, then move on the screen continuously 

indicating the proper position of the reference mark during the relocation. The operator (or other sensor) will always have a reference, will always be able to judge the relative position of the micro-manipulator, and will be able to make corrections throughout the loop. More frequent corrections should result in smallcr errors and faster corrections.

\subsection{Surface Modeling}

The tests presented in this report presumed the controllers had no information about the shape of the surface. Information on the curvature could prompt the controller to make adjustments before an error becomes apparent. Even when the observations and corrections are not perfect, the remaining error that must be corrected by the operator would likely be reduced.

\section{References}

[1] Carderock Division, Naval Surface Warfare Center, SOL N00167-97-SS-R1, "Mechanical Ship Híull Paint Application System For Usc in Drydock", Commerce Business Daily, April 10, 1997.

[2] T. Yoshikawa, K. Hosoda, T. Doi, H. Murakami, "Dynamic Trajectory Tracking Control of Flexible Manipulator by Macro-Micro Manipulator System", Proc of ICRA, pp. 1804-1809, 1994.

[3] T. Yoshikawa, K. Harada, A. Matsumoto, "Hybrid Position/Forcc Control of Flexible-Macro/RigidMicro Manipulator System", IEEE Transactions on Robotics and Automation, Vol. 12, No. 4, Aug 1996.

[4] O. Khatib, "Reduccd Effective Incrtia in Macro/Mini Manipulator Systems", Proceedings of ACC. pp. $2140-2147(1988)$.

[5] K. Nagai, T. Yoshikawa, "Impedance Control of Redundant Macro-Micro Manipulators", Proc. of Int'l Conf on Intelligent Robots and Systems, pp. 1438-1445, 1994.

[6] A. Sharon, N. Hogan, D. Hawitt, "High Bandwidth Force Regulation and Inertia Reduction Using a Macro/Micro Manipulator System", IEEE ICRA, PP. 126-132, 1988.

[7] K. Nagai, Y. Nakagawa, S. IWASA, K. Ohno, "Development of a Redundant Macro-Micro Manipulator and Contour Tasks Utilizing its Compliant Motion". Proc. of Int'l Conf on Intelligent Robots and Systcms, vol. 1, pp. 279-284, 1997.

[8] T. Narikiyo, H. Nakane, T. Akuta, N. Mohri, N. Saito, "Control System Design, for Macro/Micro Manipulator with Application to Electrodischargc Machining", Intclligent Robots and Systems, pp 1454-1460, 1994.

[9] J. Albus, H. McCain. R. Lumia, "NASA/NBS Standard Reference Model for Tclerobot Control System Architecture (NASREM)", NIST Tcchnical Report 1235, Gaithersburg, MD 1989.

[10] R. Norcross , "Trajectory Considerations for the Automatcd Paint Application, Containment and Treatment System (APACTS)", NIST Technical Report 6326, Gaithersburg, MD 1999. 


